\title{
Time-resolved Spectroscopy of Interparticle Coulombic Decay Processes
}

\author{
Elke Fasshauer * and Lars Bojer Madsen \\ Department of Physics and Astronomy, Aarhus University \\ Ny Munkegade 120, 8000 Aarhus, Denmark
}

(Dated: March 18, 2020)

\begin{abstract}
We report theory for time-resolved spectator resonant Interparticle Coulombic Decay (ICD) processes. Following excitation by a short extreme ultraviolet pulse, the spectrum of the resonant ICD electron develops. Strong-field ionization is imagined to quench the decay at different time delays and to initiate regular ICD.In this latter process, the ICD electron signal can be measured without interference effects. The typical lifetimes of ICD processes allow for the observation of oscillations of the time- and energy-differential ionization probability. We propose to utilize this oscillation to measure lifetimes of electronic decay processes.
\end{abstract}

\section{INTRODUCTION}

Interparticle Coulombic Decay (ICD) [1, 2] is an electronic decay process of ionized systems consisting of at least two weakly bonded units, be it atoms, molecules or clusters. ICD has been observed in multiple systems like noble gas clusters [3 11] and clusters of different solvent molecules like water [4, 5, 12, or ammonia [13-16. It allowed to explain the repair mechanism of the enzyme photolyase [17] and was used to establish a more efficient double ionization strategy [18. ICD is furthermore discussed as a source of slow electrons, which are most efficient in damaging the DNA after exposure to high energy radiation or radioactive materials in the human body 1924 . In this work, we shed light on this fundamental process by offering a time-resolved perspective directly of the electron dynamics.

Due to developments in creating short pulses in the extreme ultraviolet (XUV) and x-ray domain [26] a time-dependent investigation of ICD processes should be within reach. Fano profiles of a much faster autoionization (AI) process were recently measured [27, 28. A few time-resolved investigations of ICD have already been performed theoretically and experimentally, where the ions produced in the process were measured [29 36]. However, electrons can be measured with higher energy resolution than ions and grant direct access to the electron dynamics and interference effects during the process. In this work, we will therefore focus on the evolution of the time- and energy-differential ionization probability, propose how it might be possible to measure this quantity in experiment, and discuss how decay lifetimes can be determined from the time-resolved signal.

In brief, the ICD process starts from a unit $A$, which is ionized in the inner valence shell. This vacancy is filled by an electron from the same unit and the excess energy is simultaneously transferred to a neighbouring unit $B$. The latter is consequently ionized by emission of the ICD electron. The two positively charged units undergo a Coulomb explosion (see Fig. 1). A related process is

\footnotetext{
* elke.fasshauer@gmail.com
}

initiated by an excitation of unit $A$. This resonant ICD (RICD) can be characterized by the behaviour of the excited electron: it either participates in the decay process or not. The respective processes are called participator RICD (pRICD) 37] and spectator RICD (sRICD) 3742. In this work, we will focus on the sRICD signal: Unit $A$ is excited from the inner valence. The vacancy is then filled by an electron from the valence and the excess energy is used to ionize the neighbouring unit $B$ (see Fig.11). This process is usually characterized by lifetimes of several tens to hundreds of femtoseconds. After the sRICD process, the excited unit $A$ decays via fluorescense within a few nanoseconds. AI and pRICD are competing decay channels and their effect on the sRICD signal is taken into account in the theory developed below.

We propose to initiate a sRICD process by exciting with a short XUV pulse. The system will then decay under emission of an sRICD electron. This is illustrated in Fig. 1 for electronic energies and resonance parameters corresponding to the neon dimer after an excitation to the Ne $2 s^{-1} 5 p$ state. At a later time $t_{s}$, a second short and intense infrared (IR) laser pulse quenches the sRICD process by ionization. By varying the time delay $t_{s}$ one should be able to observe the time-dependent formation of the sRICD signal, as illustrated to the right in Fig. 1 1 before the second laser pulse, by pump-probe spectroscopy similar to the AI process measured in Ref. [27. At the same time, the proposed quenching would initiate an ICD process involving the excited ion $A^{+*}$. Due to the strong-field ionization initiating this latter process, the signals are well-separated in energy. In this paper we provide the basic formulation for a purely electronic solution upon which more complex scenarios, including nuclear dynamics, will be built in future work.

\section{THEORY}

The relevant property for the description of the time evolution of the ICD processes is the time- and energy-differential ionization probability obtained from the time-dependent wavefunction $|\Psi(t)\rangle$ by $P\left(E_{\text {kin }}, t\right)=$ $\sum_{i}\left|\left\langle E_{i} \mid \Psi(t)\right\rangle\right|^{2}$. Here $\left|E_{i}\right\rangle$ denotes a continuum state with 


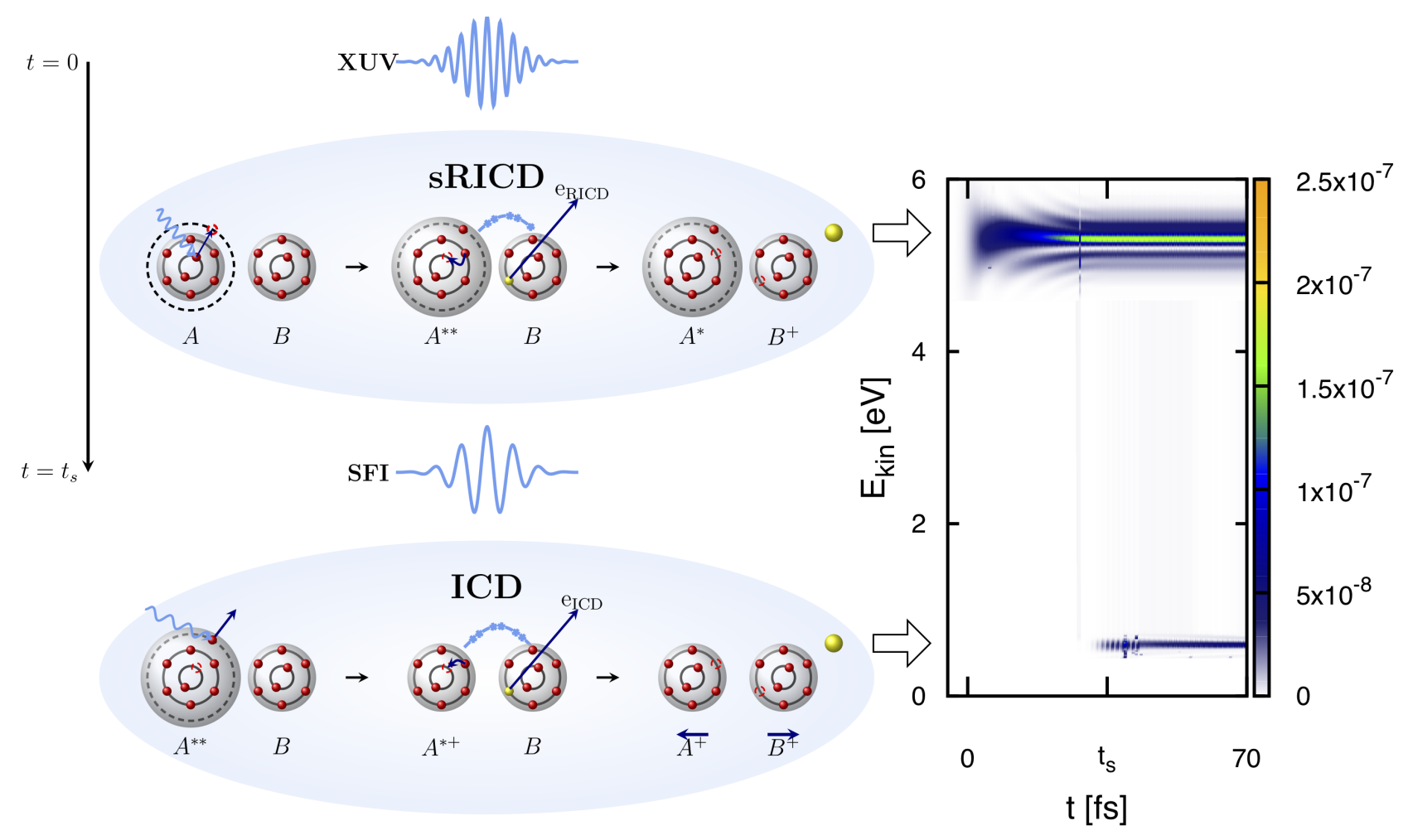

FIG. 1. Illustration [25] of the considered process. At $t=0$, a spectator Resonant Interparticle Coulombic Decay (sRICD) is initiated by XUV excitation. At a later time $t_{s}$, which in this example plot is $35 \mathrm{fs}$, a second strong infrared laser pulse quenches the sRICD process by ionization and thereby initiates ICD. The signals of the respective electrons with kinetic energies $E_{\text {kin }}$ are well separated. While the sRICD appears from the start of the XUV pulse centered at $t=0$, the ICD signal appears after the second pulse. This approach allows for a time-resolved measurement of the sRICD signal and an interference free measurement of the ICD signal. sRICD: Unit $A$ is excited from the inner valence shell. The created vacancy is filled with an electron from the outer valence and the excess energy is simultaneously transferred to $B$, which emits the sRICD electron. ICD: The initial state is created by removing the excited electron in $A$. It is then filled by an electron of the outer valence of $A$ while the excess energy is transferred to $B$, which consequently emits the ICD electron. The two units are both positively charged and therefore undergo Coulomb explosion. The panel to the right illustrates typical time and energy resolved traces of sRICD and ICD electrons (see text).

energy $E_{i}$, which entails both the kinetic energy $E_{\text {kin }}$ of the emitted electron and the energy of the final cationic state $i$. These continuum states are orthogonal to all bound states of the system and amongst each other. Because the signals of the competing decay channels do not overlap, we can and will focus on the SRICD signal. Atomic units are used throughout unless stated otherwise.

We obtain the wavefunction of the system by solving the time-dependent Schrödinger equation $i \partial_{t}|\Psi(t)\rangle=$ $H(t)|\Psi(t)\rangle$ for a model system consisting of the ground state $|G\rangle$, the resonant state $|R\rangle$ for the sRICD and the resonant state $|I\rangle$ for the ICD process, as well as three sets of continuum states characterized by the respective final state energy of the decay process. All parameters describing a general resonant state are designated by the index $r$. The Hamiltonian consists of the single configuration Hamiltonian $H_{0}$, the residual configuration interaction operators $V_{\mathrm{RICD}}$ and $V_{\mathrm{ICD}}$ as well as $V_{o}$ for the com- peting autoionization and pRICD decay channels, and the operator $H_{X}(t)$ of the exciting XUV pulse:

$$
H(t)=H_{0}+V_{\mathrm{sRICD}}+V_{o}+V_{\mathrm{ICD}}+H_{X}(t) .
$$

The effect of the ionizing infrared pulse is modelled by terminating the sRICD and starting the ICD process at the time of the second pulse $t_{s}$. We will come back to this point below.

\section{A. Description of the RICD process}

The sRICD process we intend to describe in a fully time-dependent manner is schematically illustrated in Fig. 2 Starting from the ground state $|G\rangle$ we excite the system with an XUV pulse with a mean photon energy $\Omega$ into the resonant state $|R\rangle$ with the corresponding energy $E_{R}$. This resonant state can then under emission of an electron either decay to the continuum state charac- 


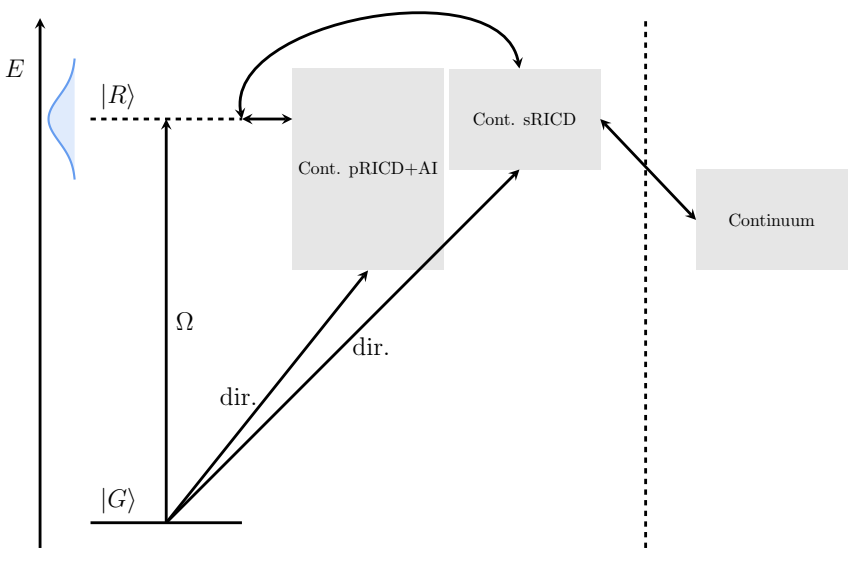

FIG. 2. Schematic illustration of the energy levels involved in the RICD process. The laser pulse with mean photon energy $\Omega$ excites the system into the resonant state $|R\rangle$ with energy $E_{R}$. The resonant state couples to two continua (denoted Cont. in the figure), one characterized by the sRICD final state, the other characterized by the pRICD and AI final state. Both channels have partial lifetimes in the order of femtoseconds. The continuum state of the sRICD process can then couple to other continuum states by emission of a photon. This fluorescence process has a lifetime $\tau_{\text {fl. }}$, which typically is in the order of nanoseconds and therefore much larger than the timescales of interest here. Alternatively, all continuum states can be reached directly by simulateneously ionizing and exciting in case of the sRICD or single ionization in case of the pRICD and AI. All couplings to the continuum via spontaneous radiative transitions are ignored in this work.

terized by a final state of the SRICD $\left|E_{s R}\right\rangle$ with a partial lifetime of $\tau_{s R}$ or to a continuum state characterized by the shared final state of pRICD and autoionization (AI) decay $\left|E_{o}\right\rangle$ with a different partial lifetime $\tau_{o}$. The continuum state of the sRICD couples to other continuum states under emission of a photon. This fluorescence process is usually several orders of magnitudes slower than the electronic decay process and is therefore ignored in this work. Alternatively to arriving in the continuum via the decaying resonant state, a simultaneous direct excitation and ionization in the case of sRICD or a direct ionization in case of the pRICD and AI channel, is also possible, which is denoted "dir" in the figure.

We assume low field strengths of the XUV pulse and therefore use first-order perturbation theory to describe its interaction with the system. Hence, the wavefunction evolves according to

$$
|\Psi(t)\rangle=\tilde{U}\left(t, t_{0}\right)\left|G\left(t_{0}\right)\right\rangle-i \int_{t_{0}}^{t} \mathrm{~d} t^{\prime} \tilde{U}\left(t, t^{\prime}\right) H_{X}\left(t^{\prime}\right)\left|G\left(t^{\prime}\right)\right\rangle .
$$

Here, $\tilde{U}\left(t, t_{0}\right)$ is the time evolution operator from a time $t_{0}$ until time $t$ of the unperturbed system pertaining to the first four terms on the right hand side of Eq. 11.

We are free to choose an energy reference and therefore set $E_{G}=0$. This conveniently removes the timedependence from the ground state, since $\left|G\left(t_{0}\right)\right\rangle=$ $\exp \left\{-i E_{G} t_{0}\right\}|G\rangle=|G\rangle$.

We introduce approximations for the time-evolution operator $\tilde{U}\left(t, t^{\prime}\right)$ appearing in the last term of Eq. 22. After the system has interacted with the XUV via $H_{X}\left(t^{\prime}\right)$ at time $t^{\prime}$, the system decays via the configuration interaction $V$. In this general discussion, $V$ refers to the sum of all configuration interaction terms of Eq. (1). We will specify the explicit form of $V$ for the different cases below. In the continuum we neglect the Coulomb interaction for simplicity, which leads to the time evolution operator

$$
\tilde{U}\left(t, t^{\prime}\right)=U_{0}\left(t, t^{\prime}\right)-i \int_{t^{\prime}}^{t} \mathrm{~d} t^{\prime \prime} U_{0}\left(t, t^{\prime \prime}\right) V \tilde{U}\left(t^{\prime \prime}, t^{\prime}\right),
$$

where $U_{0}\left(t, t^{\prime}\right)$ is the free-particle time-evolution operator.

By inserting Eq.(3) into Eq.(2) we arrive at

$$
\begin{aligned}
|\Psi(t)\rangle= & \tilde{U}\left(t, t_{0}\right)|G\rangle \\
& -i \int_{t_{0}}^{t} \mathrm{~d} t^{\prime} U_{0}\left(t, t^{\prime}\right) H_{X}\left(t^{\prime}\right)|G\rangle \\
& -\int_{t_{0}}^{t} \mathrm{~d} t^{\prime} \int_{t^{\prime}}^{t} \mathrm{~d} t^{\prime \prime} U_{0}\left(t, t^{\prime \prime}\right) V \tilde{U}\left(t^{\prime \prime}, t^{\prime}\right) H_{X}\left(t^{\prime}\right)|G\rangle .
\end{aligned}
$$

The time-evolution operator $\tilde{U}\left(t^{\prime \prime}, t^{\prime}\right)$ in the last integral describes the contribution of the Hamiltonian $H_{0}+V$. This is equivalent to the Hamilton operator used in Fano's description [43, of a decaying resonant state and below we therefore add the subscript $F$ to the time-evolution operator and remove the tilde.

We now project on a continuum state $\left|E_{s R}\right\rangle$ characterized by the sRICD final state energy of the ionized system and the kinetic energy of the emitted electron. The first term vanishes, because of the orthogonality between bound and continuum states, and we obtain

$$
\begin{aligned}
\left\langle E_{s R} \mid \Psi(t)\right\rangle= & -i \int_{t_{0}}^{t} \mathrm{~d} t^{\prime}\left\langle E_{s R}\left|U_{0}\left(t, t^{\prime}\right) H_{X}\left(t^{\prime}\right)\right| G\right\rangle \\
& -\int_{t_{0}}^{t} \mathrm{~d} t^{\prime} \int_{t^{\prime}}^{t} \mathrm{~d} t^{\prime \prime}\left\langle E_{s R}\right| U_{0}\left(t, t^{\prime \prime}\right) V \\
& \times U_{F}\left(t^{\prime \prime}, t^{\prime}\right) H_{X}\left(t^{\prime}\right)|G\rangle .
\end{aligned}
$$

The time-evolution operator $U_{0}\left(t, t^{\prime}\right)$ solves the timedependent Schrödinger equation for the Hamiltonian $\vec{p}^{2} / 2$. The corresponding wavefunctions with wavevector 
$\vec{k}$ are $\left|\Psi_{\vec{k}}^{0}(t)\right\rangle=|\vec{k}\rangle \exp \left[-i \int_{-\infty}^{t} \mathrm{~d} t^{\prime} \frac{1}{2} \vec{k}^{2}\right]$. Another way of formulating a time-evolution operator is by a projection from basis functions at time $t^{\prime}$ to such of time $t$ :

$$
U_{0}\left(t, t^{\prime}\right)=\int \mathrm{d} \vec{k}\left|\Psi_{\vec{k}}^{0}(t)\right\rangle\left\langle\Psi_{\vec{k}}^{0}\left(t^{\prime}\right)\right|
$$

At this point we only consider the kinetic energy of the emitted electron and can therefore write the projection of a general continuum state $\langle E|$ on the free-particle timeevolution operator in the following way:

$$
\begin{aligned}
\langle E| U_{0}\left(t, t^{\prime}\right) & =\langle E| \exp \left[i \Phi_{0}\left(E, t, t^{\prime}\right)\right] \\
& =\langle E| \exp \left[-i \int_{t^{\prime}}^{t}\left(\frac{k^{2}}{2}+E_{\text {fin }}\right) \mathrm{d} t^{\prime \prime \prime}\right],
\end{aligned}
$$

where $k^{2} / 2$ is the kinetic energy relative to the final state $E_{\text {fin }}$. The interaction $V$ between a general resonant state $|r\rangle$ coupling to one of the continuum states is given by

$$
V=\int \mathrm{d} E^{\prime}\left|E^{\prime}\right\rangle V_{E^{\prime} r}\left\langle r\left|+\int \mathrm{d} E^{\prime}\right| r\right\rangle V_{r E^{\prime}}\left\langle E^{\prime}\right| .
$$

For two different final states, and therefore two continua, it reads

$$
\begin{aligned}
V= & \int \mathrm{d} E_{1}^{\prime}\left|E_{1}^{\prime}\right\rangle V_{E_{1}^{\prime} r}\left\langle r\left|+\int \mathrm{d} E_{1}^{\prime}\right| r\right\rangle V_{r E_{1}^{\prime}}\left\langle E_{1}^{\prime}\right| \\
& +\int \mathrm{d} E_{2}^{\prime}\left|E_{2}^{\prime}\right\rangle V_{E_{2}^{\prime} r}\left\langle r\left|+\int \mathrm{d} E_{2}^{\prime}\right| r\right\rangle V_{r E_{2}^{\prime}}\left\langle E_{2}^{\prime}\right| .
\end{aligned}
$$

This case is, e.g., relevant for systems that can not only decay via sRICD but also via autoionization or pRICD, which both yield a singly ionized system, which is the case that we investigate. At the same time, the initiating energy of the XUV laser pulse will be chosen too low to initiate an ICD process. Hence, $V=V_{\text {sRICD }}+V_{o}$ in our case.

We also insert the resolution of the identity between the Fano time-evolution $U_{F}\left(t^{\prime \prime}, t^{\prime}\right)$ operator, to be specified below, and the Hamilton operator of the XUV field using

$$
\begin{aligned}
\mathbb{1} & =|R\rangle\left\langle R\left|+\int \mathrm{d} E_{1}^{\prime}\right| E_{1}^{\prime}\right\rangle\left\langle E_{1}^{\prime}\left|+\int \mathrm{d} E_{2}^{\prime}\right| E_{2}^{\prime}\right\rangle\left\langle E_{2}^{\prime}\right| \\
& =|R\rangle\left\langle R\left|+\int \mathrm{d} E_{s R}^{\prime}\right| E_{s R}^{\prime}\right\rangle\left\langle E_{s R}^{\prime}\left|+\int \mathrm{d} E_{o}^{\prime}\right| E_{o}^{\prime}\right\rangle\left\langle E_{o}^{\prime}\right| .
\end{aligned}
$$

By suppressing all dependences on angular momentum and using Eqs. (5), (10) and $(12)$ we arrive at an amplitude of the sRICD process for the resonance $|R\rangle$ consisting of four terms:

$$
\begin{aligned}
\left\langle E_{s R} \mid \Psi(t)\right\rangle= & -i \int_{t_{0}}^{t} \mathrm{~d} t^{\prime}\left\langle E_{s R}\left|U_{0}\left(t, t^{\prime}\right) H_{X}\left(t^{\prime}\right)\right| G\right\rangle-\int_{t_{0}}^{t} \mathrm{~d} t^{\prime} \int_{t^{\prime}}^{t} \mathrm{~d} t^{\prime \prime}\left\langle E_{s R}\left|U_{0}\left(t, t^{\prime \prime}\right)\right| E_{s R}\right\rangle V_{E R}\left\langle R\left|U_{F}^{R}\left(t^{\prime \prime}, t^{\prime}\right)\right| R\right\rangle\left\langle R\left|H_{X}\left(t^{\prime}\right)\right| G\right\rangle \\
& -\int_{t_{0}}^{t} \mathrm{~d} t^{\prime} \int_{t^{\prime}}^{t} \mathrm{~d} t^{\prime \prime} \int \mathrm{d} E_{s R}^{\prime}\left\langle E_{s R}\left|U_{0}\left(t, t^{\prime \prime}\right)\right| E_{s R}\right\rangle V_{E R}\left\langle R\left|U_{F}^{R}\left(t^{\prime \prime}, t^{\prime}\right)\right| E_{s R}^{\prime}\right\rangle\left\langle E_{s R}^{\prime}\left|H_{X}\left(t^{\prime}\right)\right| G\right\rangle \\
& -\int_{t_{0}}^{t} \mathrm{~d} t^{\prime} \int_{t^{\prime}}^{t} \mathrm{~d} t^{\prime \prime} \int \mathrm{d} E_{o}^{\prime}\left\langle E_{s R}\left|U_{0}\left(t, t^{\prime \prime}\right)\right| E_{s R}\right\rangle V_{E R}\left\langle R\left|U_{F}^{R}\left(t^{\prime \prime}, t^{\prime}\right)\right| E_{o}^{\prime}\right\rangle\left\langle E_{o}^{\prime}\left|H_{X}\left(t^{\prime}\right)\right| G\right\rangle
\end{aligned}
$$

In these expressions, $U_{0}\left(t, t^{\prime}\right)$ is the free particle time evolution operator, whose action was specified in Eq. (8). $V_{E R}=\langle E|V| R\rangle=\sqrt{\Gamma_{s R} /(2 \pi)}$ is related to the partial sRICD decay rate of the resonant state $\Gamma_{s R}$ and $U_{F}^{R}\left(t^{\prime \prime}, t^{\prime}\right)$ is the Fano time-evolution operator, which is specific to the resonant state $|R\rangle$. The variables of the other, competing, processes are designated with the index $o$. The total decay width of the RICD resonant state is given by $\Gamma_{R}=\Gamma_{s R}+\Gamma_{o}$.

The terms of Eq. (13) are linked to the different path- 
ways shown in Fig. 22 as follows. The first term of Eq. 13 describes the direct excitation and ionization to the continuum state, while the second term describes the excitation from the ground state to the resonant state followed by a decay to the continuum state. The third, indirect, term describes the direct excitation and ionization to the continuum state related to the SRICD final state, which couples to the resonant state, which then again decays into the continuum state. The last term is similar to the indirect term, but couples to the continuum of the competing pRICD and AI channels. Due to a different model system including only one final state, the latter term is not present in Eq. (11) of Ref. 44]. The interference of these different terms leads to the characteristic Fano profile 43 .

We describe the interaction between the system and the exciting XUV field in the dipole approximation. Therefore, the corresponding Hamilton operator in the length gauge is given by $H_{X}\left(t^{\prime}\right)=-\mu E_{X}\left(t^{\prime}\right)=$ $-\mu \frac{\mathrm{d}}{\mathrm{d} t^{\prime}} A_{X}\left(t^{\prime}\right) f_{X}\left(t^{\prime}\right)$, where $E_{X}\left(t^{\prime}\right)$ denotes the timedependent field strength of the XUV laser, while $\mu$ denotes the dipole operator, $A_{X}\left(t^{\prime}\right)=A_{0 X} \cos \left(\Omega t^{\prime}\right)$ is the vector potential of the laser field in the direction of the linear polarization and $f_{X}\left(t^{\prime}\right)$ is the Gaussian pulse envelope. Over the energy range of interest, we assume the transition dipole matrix element from the ground state to the continuum to be independent of the energy of the continuum state. To indicate these assumptions, we change the notation as $\left\langle E_{i}|\mu| G\right\rangle=\left\langle C_{i}|\mu| G\right\rangle$. We furthermore assume the coupling elements between the resonant and the continuum state to be real and independent of the continuum energy and therefore change the notation $V_{E_{1} R} \rightarrow V_{R}$ and $V_{E_{2} R} \rightarrow W_{R}$. Then, the Fano matrix elements, i.e., $\left\langle R\left|U_{F}^{R}\left(t^{\prime \prime}, t^{\prime}\right)\right| R\right\rangle$ and $\left\langle R\left|U_{F}^{R}\left(t^{\prime \prime}, t^{\prime}\right)\right| E^{\prime}\right\rangle$ of Eq. (13), can be solved using the projection formulation of the Fano time-evolution operator and solving the resulting integral by contour integration. Hence, we write the Fano time-evolution operator as

$$
\begin{aligned}
U_{F}\left(t^{\prime \prime}, t^{\prime}\right) & =\int \mathrm{d} \underline{E}\left|\Psi_{\underline{E}}\left(t^{\prime \prime}\right)\right\rangle\left\langle\Psi_{\underline{E}}\left(t^{\prime}\right)\right| \\
& =\int \mathrm{d} \underline{E}\left|\Psi_{\underline{E}}\right\rangle\left\langle\Psi_{\underline{E}}\right| \exp \left[-i \underline{E}\left(t^{\prime \prime}-t^{\prime}\right)\right] .
\end{aligned}
$$

The evaluation of these Fano matrix elements is given in Appendix A and for the sRICD signal we finally arrive at:

$$
\begin{aligned}
\langle E \mid \Psi(t)\rangle= & i\left\langle C_{s R}|\mu| G\right\rangle \int_{t_{0}}^{t} \mathrm{~d} t^{\prime} \exp \left[i \Phi_{0}\left(E_{s R}, t, t^{\prime}\right)\right] E_{X}\left(t^{\prime}\right) \\
+ & \left(V_{R}\langle R|\mu| G\rangle-i \pi V_{R}^{2}\left\langle C_{s R}|\mu| G\right\rangle\right. \\
& \left.-i \pi V_{R} W_{R}\left\langle C_{o}|\mu| G\right\rangle\right) \\
& \times \int_{t_{0}}^{t} \mathrm{~d} t^{\prime} \int_{t^{\prime}}^{t} \mathrm{~d} t^{\prime \prime} \exp \left[i \Phi_{0}\left(E_{s R}, t, t^{\prime \prime}\right)\right] \\
& \exp \left[-i\left(E_{R}-i \pi\left(V_{R}^{2}+W_{R}^{2}\right)\right)\left(t^{\prime \prime}-t^{\prime}\right)\right] E_{X}\left(t^{\prime}\right) .
\end{aligned}
$$

The first integral corresponds to the direct ionization process. The second integral is identical for the resonant and the two indirect terms. They have, though, different prefactors. For the case of a slowly varying envelope function $\left(\frac{\mathrm{d}}{\mathrm{d} t^{\prime}} f_{X}\left(t^{\prime}\right) \approx 0\right)$ and only considering the absorption of an XUV photon these two integrals are solved analytically for times after the exciting pulse. The direct term is given by

$$
-\frac{A_{0 X} \Omega\left\langle C_{s R}|\mu| G\right\rangle}{4} \exp \left[-i t\left(E_{\mathrm{kin}}+E_{\mathrm{fin}}\right)\right] \exp \left[-\frac{\sigma^{2}}{2}\left(E_{\mathrm{kin}}+E_{\mathrm{fin}}-\Omega\right)^{2}\right] \Re\left[\operatorname{erf}\left(\frac{1}{\sqrt{2} \sigma}\left(\frac{T_{X}}{2}+i \sigma^{2}\left(E_{\mathrm{kin}}+E_{\mathrm{fin}}-\Omega\right)\right)\right)\right]
$$

where $\Re$ denotes the real value and erf denotes the er- ror function. The resonant and indirect ionization terms without their respective prefactors are given by

$$
\begin{aligned}
& +\frac{A_{0 X} \Omega}{4\left(E_{R}-i \pi\left(V_{R}^{2}+W_{R}^{2}\right)-E_{\text {kin }}-E_{\text {fin }}\right)} \exp \left[-i t\left(E_{R}-i \pi\left(V_{r}^{2}+W_{R}^{2}\right)\right)\right] \exp \left[-\frac{\sigma^{2}}{2}\left(E_{R}-\Omega\right)^{2}\right]\left(\operatorname{erf}\left(\tau_{1, \max }\right)-\operatorname{erf}\left(\tau_{1, \min }\right)\right) \\
& -\frac{A_{0 X} \Omega}{4\left(E_{R}-i \pi\left(V_{R}^{2}+W_{R}^{2}\right)-E_{\text {kin }}-E_{\text {fin }}\right)} \exp \left[-i t\left(E_{\text {kin }}+E_{\text {fin }}\right)\right] \exp \left[-\frac{\sigma^{2}}{2}\left(E_{\text {kin }}+E_{\text {fin }}-\Omega\right)^{2}\right]\left(\operatorname{erf}\left(\tau_{2, \max }\right)-\operatorname{erf}\left(\tau_{2, \min }\right)\right)
\end{aligned}
$$


with $\tau_{1, \max }=\frac{1}{\sqrt{2} \sigma}\left(\frac{T_{X}}{2}-i \sigma^{2}\left(E_{R}-i \pi\left(V_{R}^{2}+W_{R}^{2}\right)-\Omega\right)\right)$, $\tau_{1, \text { min }}=-\frac{1}{\sqrt{2} \sigma}\left(\frac{T_{X}}{2}+i \sigma^{2}\left(E_{R}-i \pi\left(V_{R}^{2}+W_{R}^{2}\right)-\Omega\right)\right)$, $\tau_{2, \max }=\frac{1}{\sqrt{2} \sigma}\left(\frac{T_{X}}{2}-i \sigma^{2}\left(E_{\mathrm{kin}}+E_{\mathrm{fin}}-\Omega\right)\right)$ and $\tau_{2, \min }=$ $-\frac{1}{\sqrt{2} \sigma}\left(\frac{T_{X}}{2}+i \sigma^{2}\left(E_{\text {kin }}+E_{\text {fin }}-\Omega\right)\right)$. Here, $\sigma$ is the standard deviation of the Gauss distribution in time and $T_{X}$ is the duration of the exciting XUV pulse.

The time- and energy-differential ionization probability of the RICD process is the absolute square of the amplitude given in Eq. 15 and therefore a sum over 28 different terms ( 7 absolute squares and 21 mixed interference terms). Their relative contributions are determined by the Fano parameter $q=\frac{\langle R|\mu| G\rangle}{\langle C|\mu| G\rangle \pi V_{R}}$.

\section{B. Transition from the Resonant State of the RICD Process to the initial state of the ICD Process}

We propose a time-resolved measurement of the RICD process, where the quenching of the RICD process by strong field ionization with an IR pulse at time $t_{s}$ entails the initiation of an ICD process. Technically, this means a population transfer from the resonant state of the RICD process to the initial state of the ICD process. We assume the transition in time to be Gaussian shaped and that the ICD signal is unaffected by pulse shape effects. The transition is modelled as follows.

We determine the remaining population of the resonant state of the RICD process $N_{0}$ at the beginning of the second pulse $t=t_{s}-\delta t$ as:

$$
\begin{aligned}
N_{0}= & \frac{|\langle R|\mu| G\rangle|^{2}}{4} \exp \left[-\sigma^{2}\left(\Omega-E_{R}\right)^{2}\right] \\
& \times \exp \left[-\left(\Gamma_{s R}+\Gamma_{o}\right)\left(t_{s}-\delta t\right)\right] .
\end{aligned}
$$

Here, $\delta t$ is chosen as $\frac{5}{2} \sigma_{\mathrm{IR}}$ with $\sigma_{\mathrm{IR}}$ being the standard deviation of the IR pulse in time. We assume the decrease of the population $N_{R}(t)$ in the resonant state of the RICD process $|R\rangle$ to have a Gaussian shape $f_{\mathrm{IR}}(t)$ centered around the time of the second pulse:

$$
\frac{\mathrm{d} N_{R}(t)}{\mathrm{d} t}=-f_{\mathrm{IR}}(t) N_{R}(t)
$$

and therefore

$$
N_{R}(t)=N_{0} \exp \left[-\frac{\alpha}{2} \operatorname{erf}\left(-\frac{\delta t}{\sqrt{2} \sigma}, \frac{t-t_{s}}{\sqrt{2} \sigma}\right)\right] .
$$

Correspondingly, the population of the initial state $|I\rangle$ of the ICD process $N_{R}(t)$ is increased

$$
N_{I}(t)=N_{0}-N_{R}(t)
$$

The square roots of the time-dependent populations are then updated in every time step and used instead of $\langle r|\mu| G\rangle$ in the calculation of the amplitude of the ionization probability. In order to allow for a complete depopulation of the resonant state, we have chosen $\alpha=8$.

Since we assume a direct population of the resonant state of the ICD process $|I\rangle$ from the resonant state of the sRICD process $|R\rangle$ induced with a short and intense laser pulse, we assume, that the terms of Eq. (13), which are mediated by the continuum, can be neglected. After the end of the ionizing pulse the amplitude of the ICD process associated with resonance $|I\rangle$ therefore reads

$$
\langle E \mid \Psi(t)\rangle=-\int_{t_{0}}^{t} \mathrm{~d} t_{s} \int_{t_{s}+\delta t}^{t} \mathrm{~d} t^{\prime \prime}\left\langle E\left|U_{0}\left(t, t^{\prime \prime}\right)\right| E\right\rangle V_{E I}\left\langle I\left|U_{F}^{I}\left(t^{\prime \prime}, t_{s}\right)\right| I\right\rangle \sqrt{N_{I}} .
$$

\section{COMPUTATIONAL DETAILS}

In our model system, where the nuclei are kept fixed, we evaluated Eq. (13), and simulated the time-dependent build up of the Fano resonance for the sRICD process for parameters corresponding to those of the neon dimer at the equilibrium distance of $3.08 \AA$ 47] after an excitation to the Ne $2 s^{-1} 2 p^{6} 5 p_{z}$ state. The parameters are given in Table I. All simulations were performed with ELDEST [48 52, where only the outer integral over $t^{\prime}$ in Eq. 15] is solved numerically. We use the Fano parameter $q=10$. The exact $q$ value is unknown to us, but we expect it to be larger than unity due to the low probability for direct single photon ionization and excitation. Since the exact $q$-values are unknown, we assume equal values for the two continua. We have performed calculations for a range of $q$ parameters, of which a cut is shown in Fig. 3. The time of $t=93 \mathrm{fs}$ after the initiating excitation was chosen to illustrate a typical behaviour. It becomes evident that the basic features of the spectra are identical, even though the width and the amplitude of the signal differ. Therefore, the conclusions of this paper are independent of the choice of the $q$ parameter.

The exciting pulse for the main results in Figs. 1 and 5 has $A_{0 X}=5 \times 10^{8} \mathrm{~W} / \mathrm{cm}^{2}, \Omega=47.6930 \mathrm{eV}$, and a $\mathrm{FWHM}=6.1 \mathrm{fs}$ corresponding to 50 cycles. This du- 
TABLE I. Energies and lifetimes for the excited states $\mathrm{Ne} 2 s^{-1} 2 p^{6} n p-\mathrm{Ne}(n=4,5)$ undergoing RICD. The resonant energies are taken from Ref. [4], the final state energies of the sRICD Ne $2 s^{2} 2 p^{5}\left(P_{3 / 2,1 / 2}\right) n p-\mathrm{Ne} 2 p^{5}\left(P_{3 / 2,1 / 2}\right)$, of the pRICD and AI Ne $2 s^{2} 2 p^{5}\left(P_{3 / 2,1 / 2}\right)-\mathrm{Ne}$ and the ICD process were averaged to give approximations of non-relativistic energies. The lifetimes, $\tau$, of the sRICD and pRICD + AI are from Ref. [40] and the ICD lifetime are from Ref. [46].

\begin{tabular}{lrrrr}
\hline & $n=4$ & $n=5$ & $\mathrm{pRICD}+\mathrm{AI}$ & $\mathrm{ICD}$ \\
\hline$E_{r}[\mathrm{eV}]$ & 47.1230 & 47.6930 & 48.4750 \\
$E_{\mathrm{fin}}[\mathrm{eV}]$ & 41.8391 & 42.4138 & 21.6290 & 47.8688 \\
$\tau\left(2 s^{-1}\left(n p_{z}\right)^{1} \Sigma_{u}^{+}\right)[\mathrm{fs}]$ & 112 & 106 & 206 & 98 \\
\hline
\end{tabular}

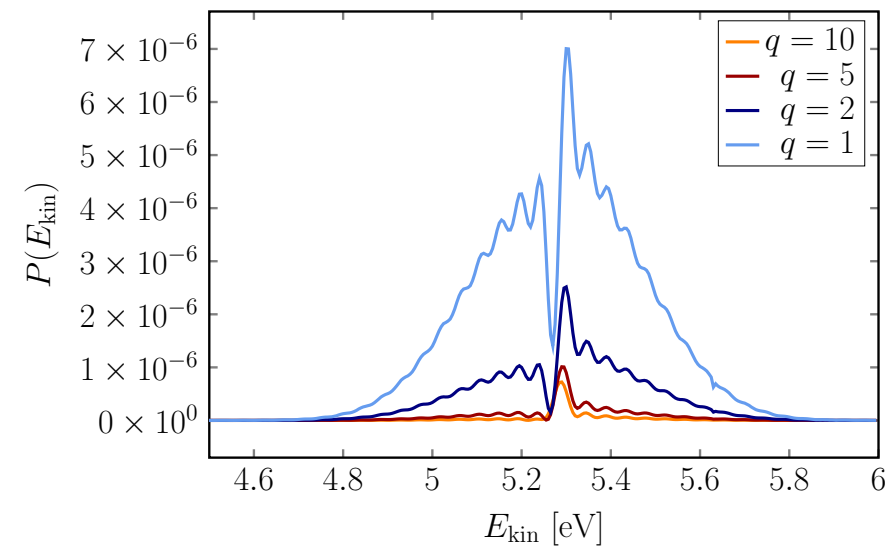

FIG. 3. Peak shape of the spectator RICD electron spectrum for 50 cycles of the exciting pulse with different values of the Fano $q$ parameter at time $t=93 \mathrm{fs}$ after the excitation at time $t=0$. The Fano profile is modified by an oscillation in energy. This oscillation stems from the interference terms of the direct term with the resonance terms of $P\left(E_{\mathrm{kin}}, t\right)$ and is described by Eq. (24). The larger $q$, the less likely the direct path and therefore, for increasing $q$, the signal is damped for a given transition dipole moment into the resonant state.

ration ensures that the bandwidth is so small that only the Ne $2 s^{-1} 2 p^{6} 5 p_{z}$ state in the neon dimer is resonantly excited.

We assumed a complete population transfer from the resonant state of the sRICD process to the resonant state of the ICD process during the second ionizing pulse over a time of $15 \mathrm{fs}$ as realized experimentally [27].

\section{RESULTS AND DISCUSSION}

In the investigation of decay processes with short laser pulses, the shortness comes with the cost of an energy broadening. As a consequence, the kinetic energy spectrum of an sRICD electron is given by a Fano profile centered around the kinetic energy, that corresponds to the energy of the resonant state, convoluted with a Gaussian centered around the kinetic energy, that would correspond to a direct excitation and ionization into the final

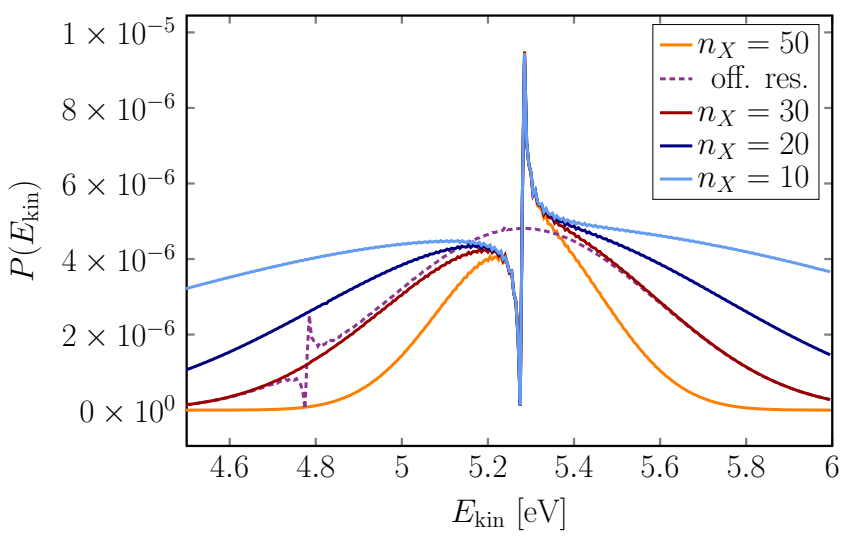

FIG. 4. Peak shape of the sRICD electron spectrum for late time $t \gg \frac{1}{\Gamma_{R}}$ after excitation of our system with electronic parameters corresponding to those of the $5 p$ state in the neon dimer depending on the number of cycles of the exciting pulse $n_{X}$. The shape can be explained by folding the Fourier transform of the exciting pulse in time with a Fano profile. In this example, the Fano parameter is $q=10$. Around the resonance energy, the peaks are identical. The width of the peak is decreased for an increased number of cycles $n_{X}$ and therefore determined by the duration of the exciting pulse. The dashed line illustrates an excitation $0.5 \mathrm{eV}$ off resonance for $n_{X}=30$.

state. Here, we show the Voigt profile part inherent to most terms of the absolute square of Eq. 15.

$$
P\left(E_{\mathrm{kin}}, \infty\right) \propto \frac{\exp \left[-\sigma_{t}^{2}\left(E_{\mathrm{kin}}+E_{\mathrm{fin}}-\Omega\right)^{2}\right]}{\left(E_{\mathrm{kin}}+E_{\mathrm{fin}}-E_{R}\right)^{2}+\frac{\Gamma_{R}^{2}}{4}}
$$

The shapes of the spectra are illustrated for different numbers of cycles $n_{X}$ for the exciting pulse in Fig. 4 for a late time $t \gg \frac{1}{\Gamma_{R}}$. There, the mean pulse energy was chosen to be on resonance and therefore, the Fano profile is centered on the maximum of the Gaussian. It is clearly seen, how the peak is narrowed with an increased number of laser cycles in the exciting pulse. In order to only excite into a single state we choose $n_{X}=50$, such that other excited states are outside $3 \sigma_{E}$ of the energy distribution of the exciting pulse. In future investigations of the interaction between several decaying resonant states, a smaller number of cycles will be appropriate.

For the case of an off-resonant excitation, the Fano profile and the Gaussian will not be centered around the same kinetic energy of the sRICD electron and the sRICD electron peak will be damped accordingly as illustrated by the dashed line of Fig. 4 for $n_{X}=30$.

By using the parameters for the neon dimer (see Table I), we simulated the time-evolution of an intial excitation at $t=0$ and quenching of the decay processes by ionization with a second laser pulse centered at the time $t=35 \mathrm{fs}$. The results are presented in Fig. 5. We will discuss its different characteristics separately.

The time-resolved spectrum shows two energyseparated signals within the chosen kinetic energy range. 


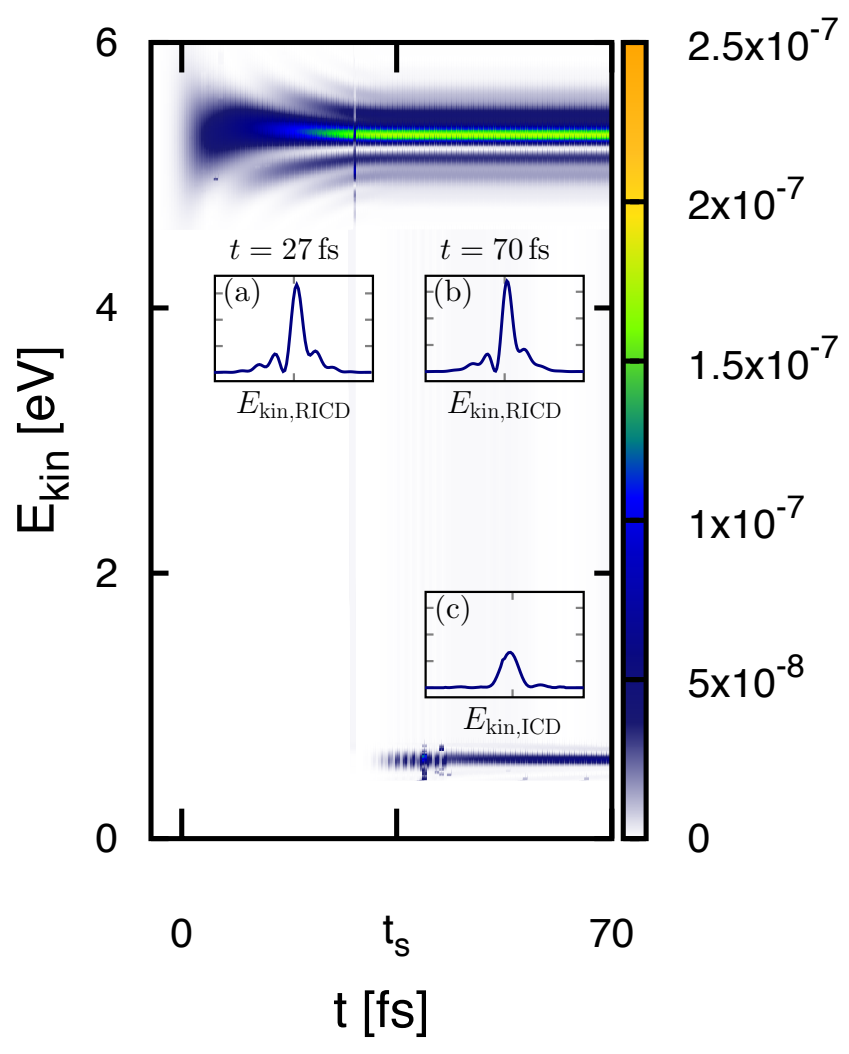

FIG. 5. Time-resolved sRICD and ICD spectrum after initial excitation at $t=0$ and quenching the sRICD at $t=35 \mathrm{fs}$. The sRICD signal centered around $5.3 \mathrm{eV}$ before the second pulse shows the buildup of a Fano profile, with an additional oscillation in energy $\cos \left[\left(E_{\mathrm{kin}}+E_{\mathrm{fin}}-E_{r}\right) t\right]$ [see (a) and text]. After depopulation of the sRICD resonant state around $t_{s}$, the signal shape remains constant [see (b)]. For the ICD signal centered around $0.6 \mathrm{eV}$ shown in (c), only the resonant term of Eq. 13 contributes, which has the shape of a Voigt profile. A pump-probe spectrum can be obtained by varying $t_{s}$

The signal of the sRICD process is initiated with the first and the signal of the ICD process by the second laser pulse, which at the same time reduces those contributions of the sRICD signal, that involve a population of the resonant state. Due to the fast depopulation of the resonant state, the signal before and after the second pulse are effectively the same [compare Fig. 5(a) and (b)]. This allows to generate a full pump-probe spectrum. The competing autoionization and pRICD process would result in much higher kinetic electron energies of about $26 \mathrm{eV}$ and can therefore unambigously be distinguished from the sRICD and ICD electrons shown in Fig. 5.

While the sRICD signal shows the buildup of a Fano profile [see Fig. [5(a)], and therefore interference effects, the ICD signal is an interference free Voigt profile [see Fig. [5(c)], because only the resonant term [see Eq. [22)] significantly contributes to it. This can be explained by the initiating process being ionization rather than excitation. In case of the ICD process, the final state is charac-

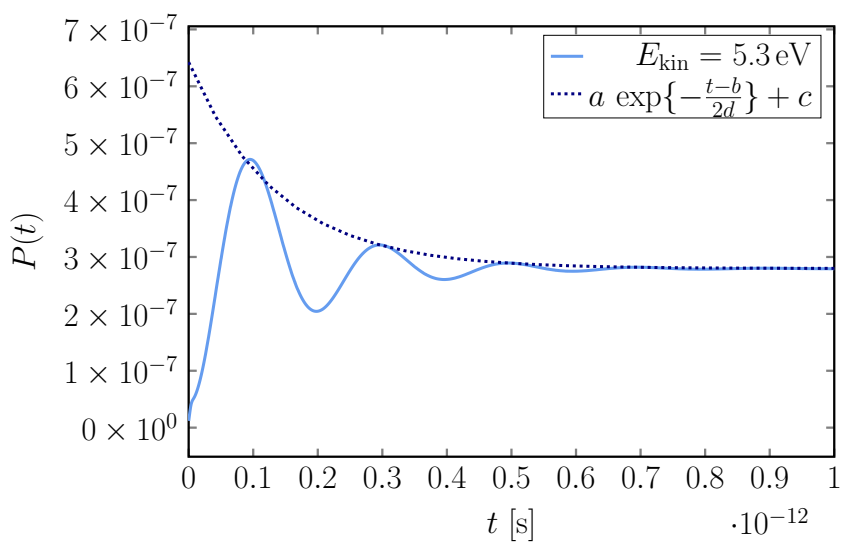

FIG. 6. Oscillation in time for observing the sRICD electron with a kinetic energy of $5.3 \mathrm{eV}$, for $n_{X}=50$ and $q=10$. This oscillation can be described by $\cos \left[\left(E_{\mathrm{kin}}+E_{\mathrm{fin}}-E_{r}\right) t\right]$. Its period is therefore depending on the kinetic energy of the electron. It is damped in time by the decay of the resonant state $\exp \left(-\Gamma_{r} t / 2\right)$. While the oscillations in energy are known and have been observed in energy, the oscillations in time require a process with a long enough lifetime to be observed around the resonance energy.

terized by a doubly ionized system. A single photon double ionization with low laser intensities is very unlikely compared to a single ionization. Hence, the corresponding Fano parameter would be very large and interference effects would have low amplitudes. At the same time, the kinetic energy distribution of the two emitted electrons can be expected to be wide and not necessarily to peak at the energy of the ICD electrons, which would further marginalize the contribution of the direct pathway. As a result, the signal shape is described by the absolute square of Eq. (22), i.e., the terms of Eq. (17).

We now turn our attention from the variation of the signals in energy to their variation in time. During the build up of the signals over time, they show oscillations in both energy and time, which are easiest seen for the ICD in the video supplementing this paper [53]. For the sRICD process, the variation in time can be obtained by solving the dominant part of the absolute square of Eq. 15 analytically. For the ICD process, the dominant part of the absolute square of Eq. (22) needs to be solved.In both cases, it can be shown that the signal is proportional to

$$
\cos \left[\left(E_{r}-E_{\mathrm{kin}}-E_{\mathrm{fin}}\right) t\right] \exp \left(-\frac{\Gamma_{r}}{2} t\right) .
$$

This equation is not specific to the decay process and therefore valid for both the sRICD and the ICD signal $(r=R, I)$. The variation in energy, illustrated in Fig. 5(a), is in agreement with Ref. [54]. As can be seen from Eq. (24), the oscillation is also visible in time as shown in Fig. 6 for a fixed kinetic energy of the sRICD electron of $E_{\text {kin }}=5.3 \mathrm{eV}$.

In order to be observed, such an oscillation requires 
long enough lifetimes of the resonant state. The comparably slow sRICD process offers the opportunity to observe and utilize it. An oscillation in time has recently been observed experimentally for an AI process [55]. In both the latter and in our case, a discrepancy between the actual and the fitted lifetime is observed. Given the analytical expressions of this work, we are able to understand this discrepancy. The kinetic energy in Fig. 6 is slightly higher than the kinetic energy corresponding to the resonant state. The further away from the resonance this energy is chosen, the shorter the period of this oscillation. The oscillation is exponentially damped by the lifetime of the decaying state. However, the dynamics of the system are more complex than shown in Eq. (24), because some terms of the resonant and indirect contributions are damped by $\exp \left(-\Gamma_{r} t\right)$ while others are damped by $\exp \left(-\Gamma_{r} t / 2\right)$, which can be seen by evaluating the time-dependence of the absolute square of Eq. 15. This behaviour is effectively only observed in the beginning of the decay, because its contribution becomes small within a short time. Therefore, a fit of the maxima at longer times, where the parts damped by $\exp \left(-\Gamma_{r} t\right)$ can be neglected, to the function $a \exp [-(t-b) / 2 d]+c$ gives a good estimate of the lifetime $d \approx \tau_{r}$. The fit in Fig. 6, where the first maximum was omitted, yields a lifetime of $68 \mathrm{fs}$. The effective overall lifetime of the excited resonant state used as input parameter for the simulations is given by $\frac{1}{\tau_{\text {eff }}}=\frac{1}{\tau_{\text {SRICD }}}+\frac{1}{\tau_{0}}$ and $\tau_{\text {eff }}=70 \mathrm{fs}$ using the parameters given in Table I The value determined from the fit is slightly lower than this effective lifetime but still matches it very well. This kind of fit can therefore be used to determine the lifetime of the electronic decay process.

\section{SUMMARY}

We have presented a time-dependent theoretical description of the SRICD and ICD processes investigated with short laser pulses for a model system with frozen nuclei. We suggest sRICD as the primary process. We then imagine that it is possible to quench the sRICD process by a strong laser pulse and thereby initiate the ICD process interference free. We have shown an oscillation of the ionization probability in time, which is general for eletronic decay processes. For processes with comparably long lifetimes, such as the ICD processes, this oscillation allows for a new way of measuring the lifetime of the underlying processes. Moreover, the basic formulation opens the door for future investigations in controlling the decay processes and to guide time-resolved experimental studies.

\section{ACKNOWLEDGEMENTS}

The research of E.F. was supported by the Villum Foundation. The research of L.B.M. was supported by the Villum Kann Rasmussen Centre of Excellence QUSCOPE - Quantum Scale Optical Processes.

\section{Appendix A: Fano matrix elements}

\section{One continuum final state}

The time-independent wavefunction $\left|\Psi_{E}\right\rangle$ is a solution to the same Hamiltonian as considered in Ref. [43]. We therefore have the same solution

$$
\left|\Psi_{\underline{E}}\right\rangle=a(\underline{E})|r\rangle+\int \mathrm{d} E^{\prime} b_{E^{\prime}}(\underline{E})\left|E^{\prime}\right\rangle
$$

with coefficients

$$
\begin{aligned}
a(\underline{E})= & -\frac{V_{r}}{\sqrt{\left(\underline{E}-E_{r}-F(\underline{E})\right)^{2}+\pi^{2} V_{r}^{4}}} \\
b_{E^{\prime}}(\underline{E})= & \frac{V_{r} a(\underline{E})}{\underline{E}-E^{\prime}} \\
& -\frac{\underline{E}-E_{r}-F(\underline{E})}{\sqrt{\left(\underline{E}-E_{r}-F(\underline{E})\right)^{2}+\pi^{2} V_{r}^{4}}} \delta\left(\underline{E}-E^{\prime}\right) .
\end{aligned}
$$

Here, $F(\underline{E})$ is a small shift in the energy position of the resonant state $|r\rangle$, which is of second order in $V$ and, which we neglect in the following.

The Fano matrix elements of Eq. (13) involving the Fano time-evolution operator can now be solved by contour integration in the negative complex half-plane, where $a_{-1}$ is the first order residue and we use that $\langle r \mid r\rangle=1,\left\langle E \mid E^{\prime}\right\rangle=\delta\left(E-E^{\prime}\right)$ and $\langle E \mid r\rangle=0$ : 


$$
\begin{aligned}
\left\langle r\left|U_{F}\left(t^{\prime \prime}, t^{\prime}\right)\right| r\right\rangle= & \int \mathrm{d} \underline{E} \exp \left[-i \underline{E}\left(t^{\prime \prime}-t^{\prime}\right)\right]|a(\underline{E})|^{2} \\
= & V_{r}^{2} \int \mathrm{d} \underline{E} \frac{\exp \left[-i \underline{E}\left(t^{\prime \prime}-t^{\prime}\right)\right]}{\left(\underline{E}-E_{r}\right)^{2}+\pi^{2} V_{r}^{4}} \\
= & V_{r}^{2} \int \mathrm{d} \underline{E} \frac{\exp \left[-i \underline{E}\left(t^{\prime \prime}-t^{\prime}\right)\right]}{\left(\underline{E}-E_{r}+i \pi V_{r}^{2}\right)\left(\underline{E}-E_{r}-i \pi V_{r}^{2}\right)} \\
= & V_{r}^{2} \exp \left[-i E_{r}\left(t^{\prime \prime}-t^{\prime}\right)\right] \int \mathrm{d} \tilde{E} \frac{\exp \left[-i \tilde{E}\left(t^{\prime \prime}-t^{\prime}\right)\right]}{\left(\tilde{E}+i \pi V_{r}^{2}\right)\left(\tilde{E}-i \pi V_{r}^{2}\right)} \\
= & 2 \pi i V_{r}^{2} \exp \left[-i E_{r}\left(t^{\prime \prime}-t^{\prime}\right)\right] a_{-1} \\
= & \exp \left[-i\left(E_{r}-i \pi V_{r}^{2}\right)\left(t^{\prime \prime}-t^{\prime}\right)\right] \\
\int \mathrm{d} E^{\prime}\left\langle r\left|U_{F}\left(t^{\prime \prime}, t^{\prime}\right)\right| E^{\prime}\right\rangle & =\int \mathrm{d} \underline{E} \int \mathrm{d} E^{\prime} \exp \left[-i \underline{E}\left(t^{\prime \prime}-t^{\prime}\right)\right] a(\underline{E}) b_{E^{\prime}}^{*}(\underline{E}) \\
& =V_{r}^{2} \int \mathrm{d} \underline{E} \frac{\left(\underline{E}-E_{r}\right) \exp \left[-i \underline{E}\left(t^{\prime \prime}-t^{\prime}\right)\right]}{\left(\underline{E}-E_{r}\right)^{2}+\pi^{2} V_{r}^{4}} \\
& =V_{r}^{2} \int \mathrm{d} \underline{E} \frac{\left(\underline{E}-E_{r}\right) \exp \left[-i \underline{E}\left(t^{\prime \prime}-t^{\prime}\right)\right]}{\left(\underline{E}-E_{r}+i \pi V_{r}^{2}\right)\left(\underline{E}-E_{r}-i \pi V_{r}^{2}\right)} \\
& =V_{r}^{2} \exp \left[-i E_{r}\left(t^{\prime \prime}-t^{\prime}\right)\right] \int \mathrm{d} \tilde{E} \frac{\tilde{E} \exp \left[-i \tilde{E}\left(t^{\prime \prime}-t^{\prime}\right)\right]}{\left(\tilde{E}+i \pi V_{r}^{2}\right)\left(\tilde{E}-i \pi V_{r}^{2}\right)} \\
& =2 \pi i V_{r}^{2} \exp \left[-i E_{r}\left(t^{\prime \prime}-t^{\prime}\right)\right] a-1 \\
& =-i \pi V_{r} \exp \left[-i\left(E_{r}-i \pi V_{r}^{2}\right)\left(t^{\prime \prime}-t^{\prime}\right)\right]
\end{aligned}
$$

\section{Two continuum final states}

with coefficients

The time-independent wavefunction $\left|\Psi_{E}\right\rangle$ is a solution to the same Hamiltonian as considered in Ref. 43. After neglecting $F(\underline{E})$ as before we therefore have the same solutions

$$
\left|\Psi_{\underline{E}}\right\rangle=a(\underline{E})|r\rangle+\int \mathrm{d} E_{1}^{\prime} b_{E_{1}^{\prime}}(\underline{E})\left|E_{1}^{\prime}\right\rangle+\int \mathrm{d} E_{2}^{\prime} b_{E_{2}^{\prime}}(\underline{E})\left|E_{2}^{\prime}\right\rangle
$$

$$
\begin{aligned}
a(\underline{E}) & =-\frac{\sqrt{V_{r}^{2}+W_{r}^{2}}}{\sqrt{\left(\underline{E}-E_{r}\right)^{2}+\pi^{2}\left(V_{r}^{2}+W_{r}^{2}\right)^{2}}} \\
b_{E_{1}^{\prime}}(\underline{E}) & =\frac{V_{r} a(\underline{E})}{\sqrt{V_{r}^{2}+W_{r}^{2}}\left(\underline{E}-E_{1}^{\prime}\right)}-\frac{\underline{E}-E_{r}}{\sqrt{\left(\underline{E}-E_{r}\right)^{2}+\pi^{2}\left(V_{r}^{2}+W_{r}^{2}\right)^{2}}} \delta\left(\underline{E}-E_{1}^{\prime}\right) \\
c_{E_{2}^{\prime}}(\underline{E}) & =\frac{W_{r} a(\underline{E})}{\sqrt{V_{r}^{2}+W_{r}^{2}}\left(\underline{E}-E_{2}^{\prime}\right)}-\frac{\underline{E}-E_{r}}{\sqrt{\left(\underline{E}-E_{r}\right)^{2}+\pi^{2}\left(V_{r}^{2}+W_{r}^{2}\right)^{2}}} \delta\left(\underline{E}-E_{2}^{\prime}\right) .
\end{aligned}
$$

The Fano integrals can now be solved by contour integration in the negative complex half-plane, where $a_{-1}$ is the first order residue and we use that $\langle r \mid r\rangle=1$, $\left\langle E \mid E^{\prime}\right\rangle=\delta\left(E-E^{\prime}\right)$ and $\langle E \mid r\rangle=0$ : 


$$
\begin{aligned}
&\left\langle r\left|U_{F}\left(t^{\prime \prime}, t^{\prime}\right)\right| r\right\rangle=\left(V_{r}^{2}+W_{r}^{2}\right) \int \mathrm{d} \underline{E} \exp \left[-i \underline{E}\left(t^{\prime \prime}-t^{\prime}\right)\right]|a(\underline{E})|^{2} \\
&=\left(V_{r}^{2}+W_{r}^{2}\right) \int \mathrm{d} \underline{E} \frac{\exp \left[-i \underline{E}\left(t^{\prime \prime}-t^{\prime}\right)\right]}{\left(\underline{E}-E_{r}\right)^{2}+\pi^{2}\left(V_{r}^{2}+W_{r}^{2}\right)^{2}} \\
&=\left(V_{r}^{2}+W_{r}^{2}\right) \int \mathrm{d} \underline{E} \frac{\exp \left[-i \underline{E}\left(t^{\prime \prime}-t^{\prime}\right)\right]}{\left(\underline{E}-E_{r}+i \pi\left(V_{r}^{2}+W_{r}^{2}\right)\right)\left(\underline{E}-E_{r}-i \pi\left(V_{r}^{2}+W_{r}^{2}\right)\right)} \\
&=\left(V_{r}^{2}+W_{r}^{2}\right) \exp \left[-i E_{r}\left(t^{\prime \prime}-t^{\prime}\right)\right] \int \mathrm{d} \tilde{E} \frac{\exp \left[-i \tilde{E}\left(t^{\prime \prime}-t^{\prime}\right)\right]}{\left(\tilde{E}+i \pi\left(V_{r}^{2}+W_{r}^{2}\right)\right)\left(\tilde{E}-i \pi\left(V_{r}^{2}+W_{r}^{2}\right)\right)} \\
&=2 \pi i\left(V_{r}^{2}+W_{r}^{2}\right) \exp \left[-i E_{r}\left(t^{\prime \prime}-t^{\prime}\right)\right] a_{-1} \\
&=\exp \left[-i\left(E_{r}-i \pi\left(V_{r}^{2}+W_{r}^{2}\right)\right)\left(t^{\prime \prime}-t^{\prime}\right)\right] \\
& \int \mathrm{d} E^{\prime}\left\langle r\left|U_{F}\left(t^{\prime \prime}, t^{\prime}\right)\right| E^{\prime}\right\rangle=\int \mathrm{d} \underline{E} \int \mathrm{d} E^{\prime} \exp \left[-i \underline{E}\left(t^{\prime \prime}-t^{\prime}\right)\right] a(\underline{E}) b_{E^{\prime}}^{*}(\underline{E}) \\
&=V_{r} \int \mathrm{d} \underline{E} \frac{\left(\underline{E}-E_{r}\right) \exp \left[-i \underline{E}\left(t^{\prime \prime}-t^{\prime}\right)\right]}{\left(\underline{E}-E_{r}\right)^{2}+\pi^{2}\left(V_{r}^{2}+W_{r}^{2}\right)^{2}} \\
&=V_{r} \int \mathrm{d} \underline{E} \frac{\left(\underline{E}-E_{r}\right) \exp \left[-i \underline{E}\left(t^{\prime \prime}-t^{\prime}\right)\right]}{\left(\underline{E}-E_{r}+i \pi\left(V_{r}^{2}+W_{r}^{2}\right)\right)\left(\underline{E}-E_{r}-i \pi\left(V_{r}^{2}+W_{r}^{2}\right)\right)} \\
&=V_{r} \exp \left[-i E_{r}\left(t^{\prime \prime}-t^{\prime}\right)\right] \int \mathrm{d} \tilde{E} \frac{\tilde{E}^{2} \exp \left[-i \tilde{E}\left(t^{\prime \prime}-t^{\prime}\right)\right]}{\left(\tilde{E}+i \pi\left(V_{r}^{2}+W_{r}^{2}\right)\right)\left(\tilde{E}-i \pi\left(V_{r}^{2}+W_{r}^{2}\right)\right)} \\
&=2 \pi i V_{r} \exp \left[-i E_{r}\left(t^{\prime \prime}-t^{\prime}\right)\right] a_{-1} \\
&=-i \pi V_{r} \exp \left[-i\left(E_{r}-i \pi\left(V_{r}^{2}+W_{r}^{2}\right)\right)\left(t^{\prime \prime}-t^{\prime}\right)\right] .
\end{aligned}
$$

[1] L. S. Cederbaum, J. Zobeley, and F. Tarantelli, "Giant Intermolecular Decay and Fragmentation of Clusters," Phys. Rev. Lett. 79, 4778 (1997).

[2] S. Marburger, O. Kugeler, U. Hergenhahn, and T. Möller, "Experimental evidence for interatomic coulombic decay in ne clusters," Phys. Rev. Lett. 90, 203401 (2003).

[3] R. Santra, J. Zobeley, L. S. Cederbaum, and N. Moiseyev, "Interatomic Coulombic Decay in van der Waals Clusters and Impact of Nuclear Motion," Phys. Rev. Lett. 85, 4490 (2000).

[4] U. Hergenhahn, "Interatomic and Intermolecular Coulombic Decay: The Early Years," J. Electron Spectrosc. Relat. Phenom. 184, 78 (2011).

[5] T. Jahnke, "Interatomic and intermolecular Coulombic decay: the coming of age story," J. Phys. B: Atomic, Molecular and Optical Physics 48, 082001 (2015).

[6] E. Fasshauer, "Non-nearest neighbour ICD in clusters," New J. Phys. 18, 043028 (2016).

[7] E. Faßhauer, N. V. Kryzhevoi, and M. Pernpointner, "Possible electronic decay channels in the ionization spectra of small clusters composed of Ar and Xe: A fourcomponent relativistic treatment," J. Chem. Phys. 133, 014303 (2010).

[8] E. Fasshauer, M. Pernpointner, and K. Gokhberg, "In- teratomic decay of inner-valence ionized states in arxe clusters: Relativistic approach," J. Chem. Phys. 138, 014305 (2013).

[9] E. Fasshauer, M. Förstel, S. Pallmann, M. Pernpointner, and U. Hergenhahn, "Using icd for structure analysis of clusters: a case study on coexpanded ne-ar clusters," New J. Phys. 16, 103026 (2014).

[10] M. Förstel, M. Mucke, T. Arion, T. Lischke, M. Pernpointner, U. Hergenhahn, and E Fasshauer, "LongRange Interatomic Coulombic Decay in ArXe Clusters: Experiment and Theory," J. Phys. Chem. C 120, 22957 (2016).

[11] E. Fasshauer, M. Förstel, M. Mucke, T. Arion, and U. Hergenhahn, "Theoretical and experimental investigation of Electron Transfer Mediated Decay in ArKr clusters," Chem. Phys. 482, 226 (2017).

[12] I. B. Müller and L. S. Cederbaum, "Ionization and double ionization of small water clusters," J. Chem. Phys. 125, 204305 (2006).

[13] N. V. Kryzhevoi and L. S. Cederbaum, "Using pH Value To Control Intermolecular Electronic Decay," Angew. Chem. Int. Ed. 50, 1306 (2011).

[14] S. D. Stoychev, A. I. Kuleff, and L. S. Cederbaum, "Intermolecular Coulombic Decay in Small Biochemically Relevant Hydrogen-Bonded Systems," J. Am. Chem. 
Soc. 133, 6817 (2011).

[15] N. V. Kryzhevoi and L. S. Cederbaum, "Nonlocal Effects in the Core Ionization and Auger Spectra of Small Ammonia Clusters," J. Phys. Chem. B 115, 5441 (2011)

[16] B. Oostenrijk, N. Walsh, J. Laksman, E. P. Mansson, Ch. Grunewald, S. L. Sorensen, and M. Gisselbrecht, "The role of charge and proton transfer in fragmentation of hydrogen-bonded nanosystems: the breakup of ammonia clusters upon single photon multi-ionization," Phys. Chem. Chem. Phys. 20, 932 (2018).

[17] P. H. P. Harbach, M. Schneider, S. Faraji, and A. Dreuw, "Intermolecular coulombic decay in biology: the initial electron detachment from fadh- in dna photolyases," J. Phys. Chem. Lett. 4, 943 (2013).

[18] V. Stumpf, K. Gokhberg, and L. S. Cederbaum, "The role of metal ions in X-ray-induced photochemistry," Nature Chemistry 8, 237 (2016).

[19] Badia Boudaïffa, Pierre Cloutier, Darel Hunting, Michael A. Huels, and Léon Sanche, "Resonant formation of dna strand breaks by low-energy (3 to $20 \mathrm{ev)} \mathrm{elec-}$ trons," Science 287, 1658-1660 (2000).

[20] E. Brun, P. Cloutier, C. Sicard-Roselli, M. Fromm, and L. Sanche, "Damage induced to dna by low-energy (0-30 ev) electrons under vacuum and atmospheric conditions," J. Phys. Chem. B 113, 10008 (2009).

[21] X. Pan, P. Cloutier, D. Hunting, and L. Sanche, "Dissociative electron attachment to dna," Phys. Rev. Lett. 90, 208102 (2003).

[22] F. Martin, P. D. Burrow, Z. Cai, P. Cloutier, D. Hunting, and L. Sanche, "Dna strand breaks induced by $0-4$ ev electrons: The role of shape resonances," Phys. Rev. Lett. 93, 068101 (2004).

[23] E. Surdutovich and A. V. Solov'yov, "Double strand breaks in DNA resulting from double ionization events," Eur. Phys. J. D: Atomic, Molecular, Optical and Plasma Physics 66, 206 (2012).

[24] E. Alizadeh, T. M. Orlando, and L. Sanche, "Biomolecular Damage Induced by Ionizing Radiation: The Direct and Indirect Effects of Low-Energy Electrons on DNA," Annu. Rev. Phys. Chem. 66, 379 (2015).

[25] E. Fasshauer, DOI:10.6084/m9.figshare.7808294.v2.

[26] Ferenc Krausz and Misha Ivanov, "Attosecond physics," Rev. Mod. Phys. 81, 163-234 (2009).

[27] A. Kaldun, A. Blättermann, V. Stooß, S. Donsa, H. Wei, R. Pazourek, S. Nagele, C. D. Ott, C. Lin, J. Burgdörfer, and T. Pfeifer, "Observing the ultrafast buildup of a fano resonance in the time domain," Science 354, 738 (2016).

[28] V. Gruson, L. Barreau, A. Jimenez-Galan, F. Risoud, J. Caillat, A. Maquet, B. Carre, F. Lepetit, J.-F. Hergott, T. Ruchon, L. Argenti, R. Taieb, F. Martin, and P. Salieres, "Attosecond dynamics through a fano resonance: Monitoring the birth of a photoelectron," Science 354, 734 (2016).

[29] A. I. Kuleff and L. S. Cederbaum, "Tracing Ultrafast Interatomic Electronic Decay Processes in Real Time and Space," Phys. Rev. Lett. 98, 083201 (2007).

[30] K. Schnorr, A. Senftleben, M. Kurka, A. Rudenko, L. Foucar, G. Schmid, A. Broska, T. Pfeifer, K. Meyer, D. Anielski, R. Boll, D. Rolles, M. Kübel, M. F. Kling, Y. H. Jiang, S. Mondal, T. Tachibana, K. Ueda, T. Marchenko, M. Simon, G. Brenner, R. Treusch, S. Scheit, V. Averbukh, J. Ullrich, C. D. Schröter, and R. Moshammer, "Time-Resolved Measurement of Interatomic Coulombic Decay in $\mathrm{Ne}_{2}$," Phys. Rev. Lett. 111,
$093402(2013)$

[31] F. Trinter, J. B. Williams, M. Weller, M. Waitz, M. Pitzer, J. Voigtsberger, C. Schober, G. Kastirke, C. Müller, C. Goihl, P. Burzynski, F. Wiegandt, T. Bauer, R. Wallauer, H. Sann, A. Kalinin, L. Ph. H. Schmidt, M. Schöffler, N. Sisourat, and T. Jahnke, "Evolution of Interatomic Coulombic Decay in the Time Domain," Phys. Rev. Lett. 111, 093401 (2013).

[32] K. Schnorr, A. Senftleben, G. Schmid, S. Augustin, M. Kurka, A. Rudenko, L. Foucar, A. Broska, K. Meyer, D. Anielski, D. Anielski, R. Boll, D. Rolles, M. Kübel, M. F. Kling, Y. H. Jiang, S. Mondal, T. Tachibana, K. Ueda, T. Marchenko, M. Simon, G. Brenner, R. Treusch, S. Scheit, V. Averbukh, J. Ullrich, T. Pfeifer, C. D. Schröter, and R. Moshammer, "Time-resolved study of ICD in Ne dimers using FEL radiation," J. Electron Spectrosc. Relat. Phenom. 204, 245 (2015).

[33] U. Frühling, F. Trinter, F. Karimi, J. B. Williams, and T. Jahnke, "Time-resolved studies of Interatomic Coulombic Decay," J. Electron. Spectrosc. Relat. Phenom. 204, 237 (2015)

[34] T. Mizuno, P. Cörlin, T. Miteva, K. Gokhberg, A. Kuleff, L. S. Cederbaum, T. Pfeifer, A. Fischer, and R. Moshammer, "Time-resolved observation of interatomic excitation-energy transfer in argon dimers," J. Chem. Phys. 146, 104305 (2017).

[35] T. Takanashi, N. V. Golubev, C. Callegari, H. Fukuzawa, K. Motomura, D. Iablonskyi, Y. Kumagai, S. Mondal, T. Tachibana, K. Nagaya, T. Nishiyama, K. Matsunami, P. Johnsson, P. Piseri, G. Sansone, A. Dubrouil, M. Reduzzi, P. Carpeggiani, C. Vozzi, M. Devetta, M. Negro, D. Facciala, F. Calegari, A. Trabattoni, M. C. Castrovilli, Y. Ovcharenko, M. Mudrich, F. Stienkemeier, M. Coreno, M. Alagia, B. Schütte, N. Berrah, O. Plekan, P. Finetti, C. Spezzani, E. Ferrari, E. Allaria, G. Penco, C. Serpico, G. De Ninno, B. Diviacco, S. Di Mitri, L. Giannessi, G. Jabbari, K. C. Prince, L. S. Cederbaum, Ph. V. Demekhin, A. I. Kuleff, and K. Ueda, "Time-Resolved Measurement of Interatomic Coulombic Decay Induced by Two-Photon Double Excitation of Ne2," Phys. Rev. Lett. 118, 033202 (2017)

[36] Qingli Jing and Lars Bojer Madsen, "Dynamics of interatomic coulombic decay in neon dimers by xuvpump-xuv-probe spectroscopy," Phys. Rev. A 99, 013409 (2019)

[37] K. Gokhberg, V. Averbukh, and L. S. Cederbaum, "Interatomic decay of inner-valence-excited states in clusters," J. Chem. Phys. 124, 144315 (2006).

[38] S. Barth, S. Joshi, S. Marburger, V. Ulrich, A. Lindblad, G. Öhrwall, O. Björneholm, and U. Hergenhahn, "Observation of resonant Interatomic Coulombic Decay in Ne clusters," J. Chem. Phys. 122, 4 (2005).

[39] T. Aoto, K. Ito, Y. Hikosaka, E. Shigemasa, F. Penent, and P. Lablanquie, "Properties of resonant interatomic coulombic decay in ne dimers," Phys. Rev. Lett. 97, 243401 (2006).

[40] S. Kopelke, K. Gokhberg, L. S. Cederbaum, and V. Averbukh, "Calculation of resonant interatomic coulombic decay widths of inner-valence-excited states delocalized due to inversion symmetry," J. Chem. Phys. 130, 144103 (2009).

[41] A. Knie, A. Hans, M. Förstel, U. Hergenhahn, P. Schmidt, Reiß Ph., Ch. Ozga, B. Kambs, F. Trinter, 
J. Voigtsberger, D. Metz, T. Jahnke, R. Dörner, A. I. Kuleff, L. S. Cederbaum, P. V. Demekhin, and A. Ehresmann, "Detecting ultrafast interatomic electronic processes in media by fluorescence," New. J. Phys. 16, $102002(2014)$

[42] A. Hans, L. B. Ltaief, M. Förstel, Ph. Schmidt, Ch. Ozga, Ph. Reiß, X. Holzapfel, C. Küstner-Wetekam, F. Wiegandt, F. Trinter, U. Hergenhahn, T. Jahnke, R. Dörner, A. Ehresmann, Ph. V. Demekhin, and A. Knie, "Fluorescence cascades evoked by resonant interatomic Coulombic decay of inner-valence excited neon clusters," Chem. Phys. 482, 165 (2017)

[43] U. Fano, "Effects of configuration interaction on intensities and phase shifts," Phys. Rev. 124, 1866-1878 (1961).

[44] Marlene Wickenhauser, Joachim Burgdörfer, Ferenc Krausz, and Markus Drescher, "Time resolved fano resonances," Phys. Rev. Lett. 94, 023002 (2005)

[45] A. Kramida, Yu. Ralchenko, J. Reader, and NIST ASD Team (2018), "Nist atomic spectra database (version 5.6.1)," (2018), online.

[46] A. Ghosh and N. Vaval, "Geometry-dependent lifetime of Interatomic coulombic decay using equation-of-motion coupled cluster method," J. Chem. Phys. 141, 234108 (2014)

[47] A. Bondi, "van der waals volumes and radii," J. Phys.
Chem. 68, 441 (1964).

[48] ELDEST version 2.0.3, Programme for the time-resolved investigation of electronic decay processes (2020), written by E. Fasshauer, DOI:10.5281/zenodo.3700062.

[49] E. Jones, T. Oliphant, P. Peterson, et al., "SciPy: Open source scientific tools for Python," (2001-).

[50] Travis E. Oliphant, "Python for scientific computing," Computing in Science \& Engineering 9, 10 (2007)

[51] K. Jarrod Millman and Michael Aivazis, "Python for scientists and engineers," Computing in Science \& Engineering 13, 9 (2011).

[52] E. Fasshauer, (2019), input file, DOI:10.6084/m9.figshare.7808348.v1.

[53] Supplementary video of the time-resolved spectrum sRICD and ICD spectrum, where the second pulse quenches the sRICD process after $100 \mathrm{fs}$.

[54] M. Wickenhauser, Ionization Dynamics of Atoms in Femto- and Attosecond Pulses, Ph.D. thesis, Vienna University of Technology (2006).

[55] D. Busto, L. Barreau, M. Isinger, M. Turconi, C. Alexandridi, A. Harth, S. Zhong, R. J. Squibb, D. Kroon, S. Plogmaker, M. Miranda, A. Jimenez-Galan, L. Argenti, C. L. Arnold, R. Feifel, F. Martin, M. Gisselbrecht, A. L'Huillier, and P. Salieres, "Time-frequency representation of autoionization dynamics in helium," J. Phys. B: At. Mol. Opt. Phys. 51, 044002 (2018). 